\title{
Implementation of LT Code with a Novel Degree Distribution
}

\author{
Qingxiao Du, Xiaoqin Song, Ying Liu \\ College of Electronic and Information Engineering, \\ Nanjing University of Astronautics and Aeronautics \\ Nanjing, China \\ Xiwangxiaodu@126.com, xiaoqing.song@163.com
}

\author{
Liping Zhao \\ Nanjing Telecommunication Technology Institute \\ Nanjing, China \\ zhaoliping63@hotmail.com
}

\begin{abstract}
It is known that Luby Transform code (LT code) is the first code fully realizing the digital fountain concept and provides an efficient scheme to transfer information over different channels. The key to make LT code work well is the degree distribution used in the encoding procedure. It determines the degree of each encoding symbol. On the basis of robust solition distribution and optimized degree distribution, a novel degree distribution which has only one parameter is proposed in this paper. Through computer simulation, the performance of LT code with the novel degree distribution is better than the robust solition distribution and sparse degree distribution. The conclusion of the research is practically valuable in improving the efficience of data distribution application.
\end{abstract}

Keywords- LT code; degree distribution; robust solition distribution; sparse degree distribution

\section{Introduction}

Reliable transmission of data over different channels has been the subject of many researches. Digital fountain [1] is an ideal protocol which guarantees both the efficiency and the reliability of the distribution of bulk data. With the academic theory became more and more mature, fountain code attracted increasing attention in many practical applications such as multicast, downloading in parallel and streaming video [2]. Digital fountain was also widely accepted.

The LT code proposed by Luby [3] was the first practical realization of fountain code. The algorithm was further analyzed and optimized so as to improve the efficiency [4]. In the practical implementations of LT code, random number generator was employed to determine the degree and neighbors of an encoding symbol. However, the key to make LT code work well is the degree distribution used in the encoding procedure. A good degree distribution should meet the following two requirements: Firstly, as few encoding symbols as possible on average are required to ensure successful recovery of source symbols; Secondly, the average degree of the encoding symbols shall be as low as possible.

In this paper, we propose a novel degree distribution scheme which is effective in both theory and practical. Through computer simulations, it is shown that the novel degree distribution algorithm performs better than the robust solition degree distribution and the sparse degree distribution. The proposed novel degree distribution can then improve the performance of LT code.

The paper is organized as follows: Section II describes the encoding and decoding principles of LT codes. Some basic degree distributions are proposed in Section III. In Section IV, a novel degree distribution is proposed. In Section $\mathrm{V}$, the performances of novel degree distribution, robust soliton distribution and sparse degree distribution are tested and compared. Finally, the conclusions are drawn in Section VI.

\section{Encoding and Decoding Principles}

In this section, a brief introduction of the encoding and decoding of LT code is provided.

\section{A. Encoding of LT code}

In LT codes, each encoding symbol is generated and independent with all the other encoding symbols. Assume that the number of source symbols is $K$ and the degree of encoding symbols is equal to $d$. follows:

The process of generating an encoding symbol is as

- Randomly choose a degree $d$ according to the degree distribution ;

- Choose uniformly at random $d$ different source symbols as neighbors of one encoding symbol;

- XOR the $d$ different source symbols which are chosen above to generate an encoding symbol.

Repeating the above procedures can generate encoding symbols one by one.

\section{B. Decoding of LT code}

The Belief Propagation (BP) algorithm of LDPC was researched in [5-6]. However, there is an important difference: the tanner graph of LDPC code includes one kind of variable node, while LT code contains two kinds of variable nodes. One is the information variable nodes which are not transmitted and have no channel information. The other is encoding variable nodes which are transmitted over the channel.

This paper is sponsored by University of preresearch funds (NS2012045) and PLA University of Science and Technology preresearch funds (20110603) and A Project Funded by the Priority Academic Program Development of Jiangsu Higher Education Institutions(PAPD). 
For each of the encoding symbols and all relative source symbols with the encoding symbol, if those symbols take XOR operation, it results in zero. Therefore, assuming that the generated matrix of LT code is $G_{K \times N}$, in which $K$ is the number of source symbols and $N$ is the number of encoding symbols, then the pseudo checking matrix is $H_{N \times(K+N)}=\left[\begin{array}{ll}G^{T} & I\end{array}\right]$, where $I$ is a unit matrix .

Let $h_{i, j}$ denotes the value of $i$ row and $j$ column in the matrix of $H$. Some parameters of BP algorithm are as follows:

$L(m)=\left\{l: h_{m, l}=1\right\}$ : the set of code positions that participate in the $m$-th check equation.

$M(l)=\left\{m: h_{m, l}=1\right\}:$ the set of check positions in which code position $l$ participates.

$q_{m, l}^{x}$ : The probability that the value of variable node $l$ is $x$, given the information obtained via the check nodes other than check node $m$.

$r_{m, l}^{x}:$ The probability that a check node $m$ is satisfied when variable $l$ is fixed to a value $x$ and given the information obtained via the variable nodes other than variable node $l$.

In the following, binary transmission over an AWGN channel is assumed. Modulated symbols $m(i)$ are transmitted over an AWGN channel and $r_{i}=m(i)+n(i)$ denote received symbols where $n(i)$ is a Gaussian white noise which is independent and obeys normal distribution $\eta\left(0, \sigma^{2}\right)$.

\section{Initialization}

Since the receiver receives only the encoding variable nodes which are transmitted over the channel, the initial information of source symbols is zero. For $l=1,2, \mathrm{~L}, K$, initialize the priori probabilities of the nodes of source symbols:

$$
p_{l}^{1}=1 / 2, \quad p_{l}^{0}=1 / 2
$$

For $l=K+1, K+2, \mathrm{~L}, K+N$, initialize the priori probabilities of the nodes of encoding symbols

$$
p_{l}^{1}=\left(1+\exp \left(-2 \mathrm{r} / \sigma^{2}\right)\right)^{-1}, p_{l}^{0}=1-p_{l}^{1}
$$

For every $(l, m)$, such that $h_{m, l}=1$

$$
q_{m, l}^{1}=p_{l}^{1}, \quad q_{m, l}^{0}=p_{l}^{0}
$$

\section{Message passing}

The source symbols and encoding symbols of LT code are considered variable node to deal with.

Step 1: Bottom-up (horizontal):

For each $l, m$, compute $\delta r_{m, l}, r_{m, l}^{0}$ and $r_{m, l}^{1}$.

$$
\begin{gathered}
\delta r_{m, l}=\prod_{l^{\prime} \in L(m) \backslash l}\left(q_{m, l^{\prime}}^{0}-q_{m, l^{\prime}}^{1}\right) \\
r_{m, l}^{1}=\left(1-\delta r_{m, l}\right) / 2, \quad r_{m, l}^{0}=\left(1+\delta r_{m, l}\right) / 2
\end{gathered}
$$

Step 2: Top-down (vertical):
For each $l, m$, update the values of $q_{m, l}^{0}, \quad q_{m, l}^{1}$ and normalize:

$$
\begin{gathered}
q_{m, l}^{1}=p_{l}^{1} \prod_{m^{\prime} \in M(l) \backslash m} r_{m^{\prime}, l}^{1}, q_{m, l}^{0}=p_{l}^{0} \prod_{m^{\prime} \in M(l) \backslash m} r_{m^{\prime}, l}^{0} \\
\alpha=1 /\left(q_{m, l}^{0}+q_{m, l}^{1}\right) \\
q_{m, l}^{0}=\alpha q_{m, l}^{0}, \quad q_{m, l}^{0}=\alpha q_{m, l}^{0}
\end{gathered}
$$

For each $l$, compute the posteriori probabilities:

$$
\begin{gathered}
q_{l}^{1}=p_{l}^{1} \prod_{m \in M(l)} r_{m, l}^{1}, q_{l}^{0}=p_{l}^{0} \prod_{m \in M(l)} r_{m, l}^{0} \\
\beta=1 /\left(q_{l}^{0}+q_{l}^{1}\right) \\
q_{l}^{1}=\beta q_{l}^{1}, \quad q_{l}^{0}=\beta q_{l}^{0}
\end{gathered}
$$

\section{Decoding judgment}

$$
\begin{aligned}
& \text { For } l=1,2, \mathrm{~L}, K+N, \text { compute } \\
& \qquad C_{l}=\operatorname{sgn}\left(q_{l}^{0}-1 / 2\right)
\end{aligned}
$$

And get $C=\left[C_{1}, C_{2}, \mathrm{~L}, C_{K+N}\right]$.

The first $K$ of $C$ are source symbols, and the remainder of $C$ are encoding symbols.

The algorithm stops.

\section{Some Basic Degree Distribution Schemes}

In practice, the key to make LT code work well is choosing a good degree distribution.

C. Ideal Solition Distribution [7]

$$
\mu_{I S D}(i)=\left\{\begin{array}{lc}
1 / K, & i=1 \\
1 / i(i-1), & i=2,3, \mathrm{~L}, K
\end{array}\right.
$$

Ideal soliton distribution ensures that all the release probabilities are identical to $1 / K$ at each subsequent step. Hence, there is one expected ripple generated at each processing step when the number of encoding symbols is equal to $K$. After $K$ processing step, the source symbols can be ideally recovered.However, ideal soliton distribution works poorly in practice.

\section{Robust Solition Distribution [7]}

Define $R=c \cdot \ln (K / \delta) \cdot \sqrt{K}, c$ and $\delta$ are two parameters. $c$ Controls the mean of degree distribution. The smaller the $c$, the greater the probability of low degrees.is the allowable failure probability of the decoder to recover the source symbols.In this distribution, we need the help of ideal soliton distribution.

$$
\begin{gathered}
\tau(i)=\left\{\begin{array}{lc}
R /(i K), & i=1,2, \mathrm{~L}, \operatorname{round}(K / R)-1 \\
R \ln (R / \delta) / K, & i=\operatorname{round}(K / R) \\
0, & \text { else }
\end{array}\right. \\
\quad \beta=\sum_{i=1}^{K} \mu_{I S D}(i)+\tau(i)
\end{gathered}
$$




$$
\mu_{R S D}(i)=\left(\mu_{I S D}(i)+\tau(i)\right) / \beta, i=1,2, \mathrm{~L}, K
$$

Robust solition distribution is not only viable but also practical. It is an improvement of the ideal solition distribution.

\section{E. Sparse Degree Distribution [8-9]}

$$
\begin{aligned}
\Omega_{1}(x)= & 0.008 x+0.494 x^{2}+0.166 x^{3}+0.073 x^{4} \\
+ & 0.083 x^{5}+0.056 x^{8}+0.037 x^{9}+0.056 x^{19} \\
+ & 0.025 x^{65}+0.003 x^{66} \\
\Omega_{2}(x)= & 0.19 x+0.34 x^{2}+0.27 x^{4}+0.13 x^{8} \\
& +0.03 x^{16}+0.01 x^{32}+0.03 x^{64}
\end{aligned}
$$

As mentioned above, $\Omega_{1}(x)$ and $\Omega_{2}(x)$ are two different sparse degree distribution schemes. The index of $x$ is the degree and the value which is in front of $x$ is the probability of the degree.

\section{F. Optimized Degree Distribution [9]}

$$
\begin{gathered}
\varphi(i)= \begin{cases}0.083, & i=1 \\
0.487, & i=2 \\
0.032, & i=100 \\
1 / i(i-1), & \text { else }\end{cases} \\
\quad \beta=\sum_{i=1}^{K} \varphi(i) \\
\mu_{O P D}(i)=\varphi(i) / \beta, \quad i=1,2, \mathrm{~L}, K
\end{gathered}
$$

The optimized degree distribution has shown outstanding performance. It also gives us some valuable references to find other forms of degree distributions which make LT code work better.

\section{A Novel Degree Distribution Scheme}

In this section, on the basis of robust solition distribution and optimized degree distribution, we proposed a novel degree distribution scheme.

\section{G. A Novel Degree Distribution}

Even more effective degree distributions forms have been achieved by some researcher. One characteristic of these degree distribution schemes is that the probability for degree one is less than the probability for degree two. This ensures that not too many redundant degree one packets are sent, resulting in more efficient transmission. However, the ideal solition distribution itself performs rather poorly, as is well known. To improve it, we take the first two degree probabilities as parameters.

The choice of the right parameter values with degree distribution is very important for a promising performance of the algothrim. Noted that the spike presented in robust solition distribution is really necessary, thus we considered a slightly modified form and an extra parameter for the probability of degree 100. Finally, we define the rest of our distribution to be the ideal solition distribution.
Let $R=c \cdot \ln (K) \cdot \sqrt{K}$, where $c$ is a constant and $c>0$. On the basis of robust solition distribution and optimized degree distribution, a novel degree distribution scheme is then proposed. The expression of the novel degree distribution is as follows:

$$
\begin{gathered}
\phi(i)= \begin{cases}0.083, & i=1 \\
0.487, & i=2 \\
0.032, & i=100 \\
R \ln (R) / K, & i=\operatorname{round}(K / R) \\
1 / i(i-1), & \text { else }\end{cases} \\
\quad \beta=\sum_{i=1}^{K} \phi(i)
\end{gathered}
$$

In the novel degree distribution, the allowable failure probability $\delta$ introduced by Luby was removed by considering the fact that this parameter is only approximation. In fact, the practical failure probability is much larger than $\delta$.

\section{Performance Evalution}

In this section, the performance of the novel degree distribution, the robust solition distribution and the sparse degree distribution were evaluated and compared. Bit error rate (BER) is employed as a measure of the performance of degree distribution schemes.

Definition of BER: Bit error rate is the ratio between the number of error bits that the decoder decodes the received bits comparing with source bits and the total number of bits.

Assume that the number of source symbols is 1000 , the channel is AWGN channel and the mode of modulation adopted is BPSK. Each point is the average value which is running 100 frames. Simulation results and analysis are as follows:

H. BER of LT code with novel degree distribution VS. SNR

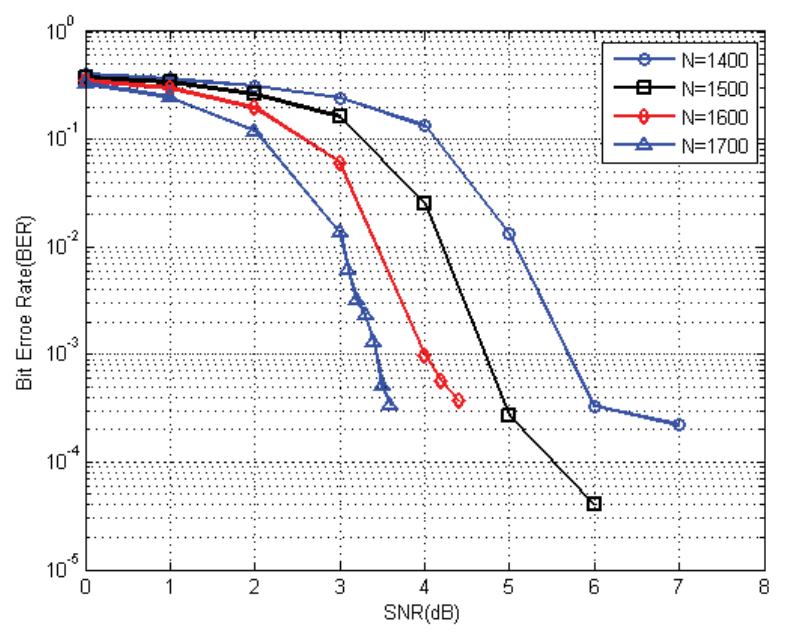

Figure 1. BER of LT Code with novel degree distribution versus SNR for transmission over AWGN channel 
In Fig. 1, the four curves demonstrate respectively the relationship of BER of LT Code with novel degree distribution versus SNR for transmission over AWGN channel when the numbers of encoding symbols received are 1400, 1500, 1600 and 1700. As can be seen from the figure, When SNR is low, even if the receiver receives more redundant encoding symbols, the BER still keep in a higher level. In case SNR $>4 \mathrm{~dB}$, however, BER drops sharply and when the received redundant symbols increases slightly, an obviously better performance was achieved.

I. BER of LT code with the novel degree distribution VS. overhead

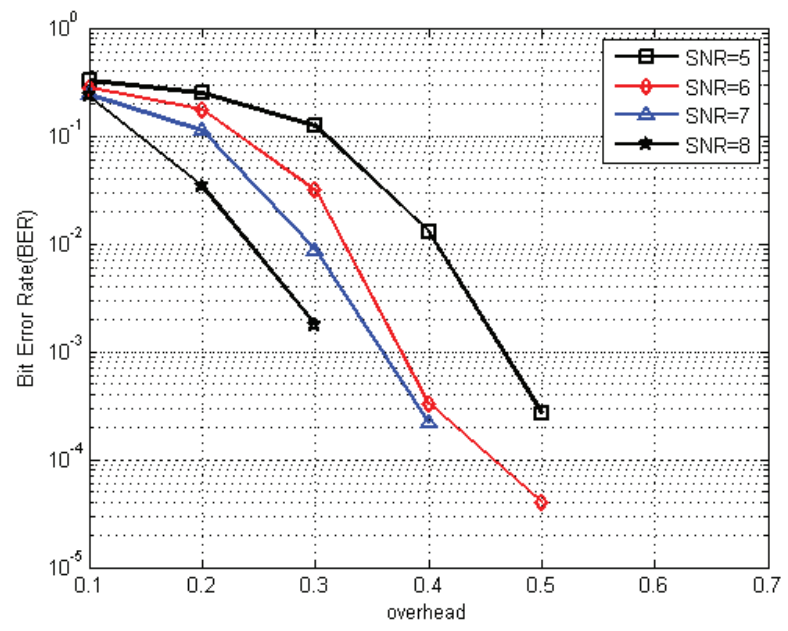

Figure 2. BER of LT Code with novel degree distribution versus overhead for transmission over AWGN channel

Fig. 2 compares the BER versus overhead in different SNR. The simulated results show that the higher the SNR, the less the redundant symbols. When overhead $<0.3$ and $\mathrm{SNR}=$ $5 \mathrm{~dB}$ (or $6 \mathrm{~dB}$ ), BER drops slowly and maintains in between $10^{-2}$ and 1 . When overhead between 0.3 and 0.5 , BER declines largely. Along with the increasing of overhead, bit error rate begins to change slowly and approach zero.

\section{J. BER of LT code with different degree distributions}

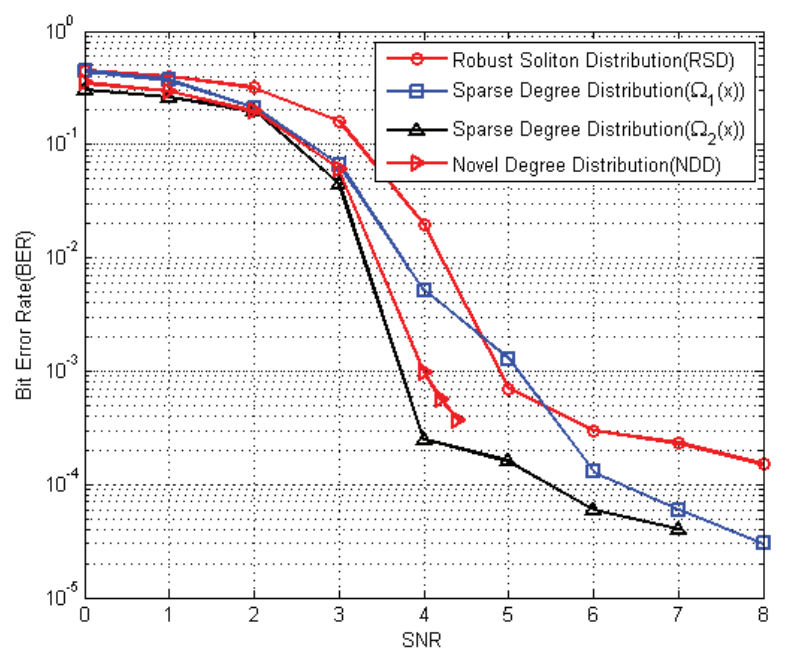

Figure 3. BER of LT Code with different degree distributions versus SNR for transmission over AWGN channel

As a comparison, we make simulation with four different degree distribution schemes in Fig. 3. When $\mathrm{SNR}<3 \mathrm{~dB}$, the performance of LT code with four different distributions is poor. When SNR is in between $3 \mathrm{~dB}$ and $4 \mathrm{~dB}$, the BER drops largely. Comparing with the robust solition distribution and the sparse degree distribution, the novel degree distribution performs obviously better than the other three schemes in higher SNR.

\section{Conclutions}

LT code is reliable and efficient in data transmission and a realization of the digital fountain. Degree distribution is important to the performance of LT code. In this paper, we proposed a novel degree distribution. Taking BER as the measure of performance, the proposed degree distribution schemes and the other three schemes were evaluated and compared through simulation. From the results of simulation, we know that the novel degree distribution performs better than robust solition distribution and sparse degree distribution. The results of the research are valuable in improving channel efficiencies of data distribution systems.

\section{REFERENCES}

[1] BYERS J W, LUBY M, MITZENMACHER M. “A digital fountain approach to reliable distribution of bulk data," Proceedings of ACM Sigcomm'98, Vancouver, Canada, Sep 1998, pp.56-67.

[2] Y.Y.Ma, D.F.Yuan, H.X.Zhang," Fountain codes and applications to reliable wireless broadcast systems, IEEE Information Theory Workshop,Chengdu,Octorber 2006, pp.66-70.

[3] M. Luby, "LT Codes," Proceedings of the 43rd Annual IEEE Symposium on Foundations of Computer Science, November 2002, pp. 271-282.

[4] Q. Zhou, L. Li, Z. Q. Chen, J. X, Zhao, “Implementation of LT codes based on chaos," Chinese Physics B, vol.17, 2008, pp. 3609-3613. 
[5] Xiaojing Feng,Wei Zhou, “Study on BP decoding algorithm of LDPC codes, " Electronic Test,NO.7 Jul.2009, pp.42-43.

[6] Robert H,Morelos-Zaragoza, “ The art of Error Correcting Coding(Second Edition), " John Wiley\&Sons,Ltd, 2006, pp.204-206.

[7] Zengqiang Chen,Qian Zhou, " Implementation of LT codes with a Revised Robust Solition Distribution by Using Kent Chaotic Map, ” 2010 International Workshop on Chaos-Fractal Theory and its Applications,2010, pp.149-153.

[8] Shokrollah A." Raptor codes, ".IEEE Transactions on Information Theory,2006,52(6):2551-2567.

[9] E. Hyytiä, T. Tirronen, J. Virtamo, "Optimal the degree distribution of LT codes with an importance sampling approach," RESIM 2006, 6th International Workshop on Rare Event Simulation, Bamberg, Germany, October,2006, pp.64-73. 